\section{PLANAR OPTICS}

\section{Ultrathin circular polarizer \\ Opt. Express 25, 14260-14269 (2017)}

A miniature circular polarizer that operates over the entire visible region and that has a thickness of just $390 \mathrm{~nm}$ has been fabricated and tested by researchers from Seoul National University in South Korea. The device is made from a stack of sets of aluminium nanoscale gratings featuring a subwavelength pitch. The orientation of each set of gratings is gradually twisted with respect to the preceding set beneath in a helix-type arrangement. As the approach relies on the non-resonant anisotropic characteristics of the grating stack, it is able to simultaneously deliver a high extinction ratio and broadband operation. The performance of stacks up to seven grating layers is investigated and as the number of gratings increases so does the extinction ratio of the polarizer, up to a value of eight across the entire visible range, but at the cost of overall transmittance due to the intrinsic loss of the aluminium. The approach is thought to be cost effective for mass production and the miniaturization it brings is thought to be potentially useful for compact mobile or wearable imaging systems. By replacing aluminium with other noble metals such as gold or silver it is possible to fabricate a design that operates in the near-infrared region. $O G$

\section{POLARITONS}

\section{Strong surprise}

Nat. Phys. http://doi.org/b89z (2017)

Polariton-based optoelectronics may turn out to be considerably more efficient than originally expected. Scientists in the US from the Massachusetts Institute of Technology, Princeton University and the University of Pittsburgh have now directly measured the strength of polariton-polariton interactions and determined that it is about two orders of magnitude stronger than previous theoretical estimates. The finding suggests that polaritonbased devices would be operating in the strongly interacting regime with an effective nonlinearity that could be larger than anticipated. Exciton-polaritons (commonly referred to as just polaritons) are a type of quasiparticle generated in a semiconductor optical microcavity - in essence, a mixed state of light and matter formed by the strong coupling between an exciton and a photon. To date, it has been difficult to accurately experimentally measure the interaction strength as it relies on being able to distinguish between polariton-polariton interactions and interactions between polaritons and excitons. The US team circumvent this problem by using a very high- $Q$ microcavity $(Q=320,000)$ so that the polaritons have a long lifetime (of the order of $>200 \mathrm{ps}$ ) and are able to propagate to an optical trap where they can be separated from free charge carriers and hot excitons. Spectral data from the centre of the trap is then used to determine the polariton interaction strength. The microcavity consists of four GaAs quantum wells sandwiched between two distributed Bragg reflectors. $O G$

\section{PHOTODETECTORS \\ Laser-defined graphene \\ Sci. Adv. 3, e1602617 (2017)}

Although many desirable properties of graphene photodetectors, such as wide bandwidth and spectral response, have been demonstrated previously, the best inorganic photodetectors are still superior in terms of the linearity of their response over a large range of optical powers. To date, the linear dynamic range (LDR) of graphene photodetectors has been limited by thermal diffusion of carriers and other effects, and this has restricted their applications in imaging. Now, Adolfo De Sanctis and colleagues from the UK and Spain have made graphene-based

\section{D MATERIALS}

\section{Negative refraction}

The unusual phenomenon of negative refraction of light is central to the realization of interesting optical devices such as superlenses and other novel schemes for guiding and imaging light. However, achieving negative refraction for light-based quasiparticles called polaritons is challenging due to the much shorter wavelengths involved, which are typically squeezed by several orders of magnitude. Now, Xiao Lin and co-workers from China, the USA and Singapore predict that negative refraction should be possible for plasmon polaritons and phonon polaritons in graphene/boron nitride heterostructures. Using the finite-element simulation method, all-angle in-plane negative refraction was numerically predicted to occur at a frequency of $22.96 \mathrm{THz}$, where the confinement factor was 195. When the graphene or the boron nitride were replaced by a hybrid layer featuring both graphene and boron nitride, the frequency at which the effect occurs could be tuned as a function of the chemical potential of the graphene layer or the thickness of the boron nitride layer. photodetectors that exhibit an LDR of $44 \mathrm{~dB}$, which they note is three orders of magnitude better than previous graphene devices. At the same time, the detectors have a broad spectral response from the ultraviolet-A to the mid-infrared and are sufficiently stable in atmospheric conditions such that encapsulation is not required. The device is made by introducing $\mathrm{FeCl}_{3}$ into the top three layers of four-layer graphene flakes. The $\mathrm{FeCl}_{3}$ in the structure was laser defined by exposing the graphene to intense green laser light (532 nm wavelength with an intensity of $15.3 \mathrm{MW} \mathrm{cm}^{-2}$ ) by raster scanning $5.5-\mu \mathrm{m}$-wide sections for $3 \mathrm{~s}$ in $0.5-\mu \mathrm{m}$ steps.

\section{NANOPHOTONICS}

\section{Thermoelectric detection}

Nat. Nanotech. http://doi.org/b89x (2017)

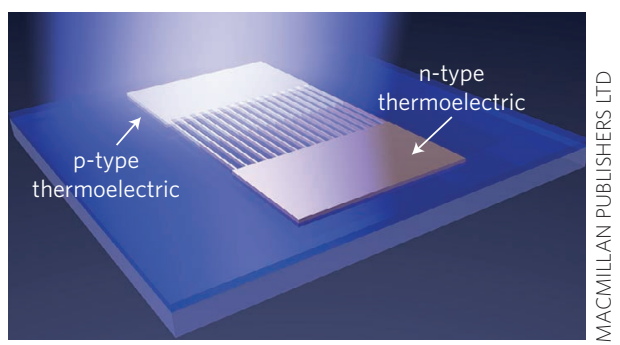

Heat generation in resonant subwavelength nanostructures is creating new opportunities for photodetector design. Now, Kelly Mauser and colleagues from the California Institute of Technology, USA have exploited resonant light absorption and the thermoelectric effect within a single suspended membrane nanostructure to realize a wavelength-tunable photodetector. The nanostructure consists of a guided mode resonance wire array on a thin, suspended, electrically insulated substrate. Made of established thermoelectric materials, bismuth telluride/antimony telluride $\left(\mathrm{Bi}_{2} \mathrm{Te}_{3}\right)$ $\left.\mathrm{Sb}_{2} \mathrm{Te}_{3}\right)$, the wires are $40 \mathrm{~nm} \times 100 \mathrm{~nm} \times 50 \mu \mathrm{m}$ and spaced $488 \mathrm{~nm}$ apart. Owing to the spatially localized absorption in resonant nanophotonic structures, localized heating of the thermoelectric material occurs, leading to large thermal gradients between the centre and the edge of the nanostructure even under unfocused optical excitation. The team have observed that the small heat capacity enables a temporal response of $337 \mu$ s, which is $10-100$ times faster than conventional thermoelectric detectors. They also show that their detectors are tunable from the visible to the mid-infrared and are capable of wavelength-specific detection, with an input power responsivity of up to $38 \mathrm{~V} \mathrm{~W}^{-1}$ and a frequency bandwidth of nearly $3 \mathrm{kHz}$.

Written by Oliver Graydon, Noriaki Horiuchi, David Pile and Rachel Won. 